\title{
Dieta de Artibeus lituratus (Olfers, 1818) (Mammalia, Chiroptera) em um fragmento florestal urbano da cidade de Londrina, Paraná, Brasil
}

\author{
Alan Deivid Pereira ${ }^{1 \star}$ \\ Nélio Roberto dos Reis ${ }^{2}$ \\ Mário Luís Orsi ${ }^{2}$ \\ Ana Paula Vidotto-Magnoni ${ }^{2}$ \\ ${ }^{1}$ Universidade Estadual de Londrina, Centro de Ciências Biológicas \\ PPG em Ciências Biológicas, AC: Biodiversidade e Conservação de Habitats Fragmentados \\ Rodovia Celso Garcia Cid, PR 445, Km 380, CEP 86.057-970, Londrina - PR, Brasil \\ ${ }^{2}$ Universidade Estadual de Londrina, Centro de Ciências Biológicas, Londrina - PR, Brasil \\ * Autor para correspondência \\ Alandeivid_bio@live.com
}

Submetido em 15/09/2018

Aceito para publicação em 12/02/2019

\section{Resumo}

Morcegos são conhecidos por sua importância na regulação dos ecossistemas e no seu envolvimento na polinização e na dispersão de sementes. Espécies de morcegos fitófagos como, por exemplo, Artibeus lituratus ocupam com frequência áreas alteradas ou até mesmo regiões urbanas. O objetivo deste estudo foi investigar a dieta de A. lituratus no campus da Universidade Estadual de Londrina, um fragmento florestal com alta interferência antrópica. Foram realizadas quatro coletas mensais, entre outubro de 2012 e setembro de 2013, utilizando quatro redes de neblina por noite. Os animais foram identificados em campo e acondicionados em sacos de algodão por 30 minutos a fim de serem obtidas amostras de fezes para a análise de sua dieta. Foram coletados 86 indivíduos, que forneceram 42 amostras fecais. Os resultados indicam que a dieta de A. lituratus é composta principalmente por frutos de Cecropiaceae e Moraceae. A amplitude de nicho trófico foi maior nos meses de março, agosto e setembro de 2013, porém não ocorreram diferenças significativas na dieta entre as estações do ano $(\mathrm{H}=0,3002$, $\mathrm{p}=0,96)$. A preferência por espécies pioneiras na dieta de A. lituratus pode ser um indicativo do seu importante papel na restauração de fragmentos florestais mesmo em ambientes urbanos.

Palavras-chave: Dispersão de sementes; Frugivoria; Restauração; Morcegos; Zoocoria

\section{Abstract}

Diet of Artibeus lituratus (Olfers, 1818) (Mammalia, Chiroptera) in an urban forest fragment in Londrina, Paraná, Brazil. Bats are known for their importance in regulating ecosystems, where they are involved in pollination and seed dispersal. Species of phytophagous bats such as Artibeus lituratus often occupy altered areas or even urban regions. The aim of this study was to investigate the diet of $A$. lituratus on the campus of the State University of Londrina, in a forest fragment with high anthropogenic influence. Four monthly collections were carried out between October 2012 and September 2013 using four mist nets per night. The animals were 
identified in the field and placed in cotton bags for half an hour to obtain fecal samples for diet analysis. A total of 86 individuals were collected, which provided $42 \mathrm{fecal}$ samples. The results indicated that the diet of $A$. lituratus is composed mainly of Cecropiaceae and Moraceae fruits. The trophic niche breadth (B') was higher in March, August and September 2013, but there were no significant differences in diet between the seasons $(H=0.3002$, $\mathrm{p}=0.96$ ). The preference for pioneer species in the diet of $A$. lituratus may be indicative of their important role in the restoration of forest fragments, even in urban environments.

Key words: Bats; Frugivory; Restoration; Seed dispersal; Zoochory

\section{Introdução}

A ordem Chiroptera é um grupo abundante e diverso, com grande representatividade em quase todo o globo. Para a região norte do Paraná, 41 espécies de morcegos foram registradas, correspondendo a $22,77 \%$ das 180 espécies de morcegos registradas para o Brasil (REIS et al., 2017). Devido a sua grande abundância, riqueza e diversidade trófica, morcegos são reconhecidamente importantes na regulação dos ecossistemas, oferecendo os mais variados serviços como polinização e dispersão de sementes (KUNZ et al., 2011).

A dispersão de sementes é importante para a manutenção e regeneração das florestas tropicais (TERBORGH; WRIGHT, 1994). Muitos trabalhos sugerem que aves e morcegos são responsáveis pela dispersão de $80 \%$ das sementes que caem ao solo (GALINDO- GONZÁLEZ et al., 2000), contudo, diferentemente das aves que evacuam empoleiradas, morcegos defecam enquanto voam, promovendo a chuva de sementes (BREDT et al., 2012).

Em florestas neotropicais, há espécies vegetais que dependem exclusivamente dos morcegos para sua dispersão (SAZIMA et al., 2003; THIES; KALKO, 2004; BREDT et al., 2012). Como destaca Lobova et al. (2009), na região Neotropical, morcegos consomem, ao menos, frutos de 546 espécies de plantas, distribuídas em 191 gêneros e 62 famílias. Bredt et al. (2012) sugerem que morcegos visitam e utilizam recursos de 720 espécies vegetais, pertencentes 82 famílias.

A família Phyllostomidae, endêmica do continente americano, destaca-se como o grupo mais versátil na exploração de alimentos entre os Chiroptera (PASSOS; GRACIOLLI, 2004). Em um ambiente pouco antropizado, a vegetação e o clima da área são fundamentais para definir quais espécies de morcegos frugívoros poderão ser encontradas na região (REIS et al., 2008). Contudo, algumas espécies de morcegos fitófagos com maior plasticidade adaptativa ocupam com frequência áreas fortemente alteradas pelo desmatamento ou até mesmo regiões urbanas (BREDT et al., 2012).

Entre as espécies que ocorrem comumente em ambientes urbanos, destaca-se Artibeus lituratus (Olfers, 1818) (ESTRADA; COATES-ESTRADA, 2002; REIS et al., 2003), um morcego considerado de grande porte, com envergadura média de $52 \mathrm{~cm}$, comprimento de antebraço de 70 a $75 \mathrm{~mm}$ e peso superior a $75 \mathrm{~g}$, caracterizado por listras brancas faciais marcantes, coloração geralmente marrom-chocolate, podendo haver alterações regionais para um tom acinzentado (PERACCHI; LIMA, 2017; PEREIRA et al., 2017).

Na região foco deste estudo, o norte do Paraná, a superfície coberta originalmente pela Floresta Estacional Semidecidual (FES), vegetação pertencente ao bioma da Mata Atlântica, foi drasticamente reduzida ao longo dos últimos 100 anos, restando apenas de 2 a $4 \%$ do ecossistema original, representado por pequenos fragmentos circundados por áreas de agricultura intensiva (SOS MATA ATLÂNTICA; INPE, 2018). Apesar da fragmentação de habitat, espécies da família Phylostomidae são comuns nos pequenos fragmentos como o Horto Florestal do campus da Universidade Estadual de Londrina, com 10 ha.

Reconhecendo o papel dos morcegos na dispersão de sementes e, consequentemente, no auxílio na recuperação de áreas degradadas, o presente trabalho descreve os itens alimentares consumidos por $A$. lituratus em um fragmento florestal situado no campus da Universidade Estadual de Londrina. 


\section{Material e Métodos}

\section{Área de estudo}

O Campus da Universidade Estadual de Londrina (UEL) $\left(23^{\circ} 19^{\prime} 19^{\prime \prime}\right.$ e e $\left.51^{\circ} 12^{\prime} 04^{\prime \prime} \mathrm{W}\right)$ está situado na região sudoeste do município de Londrina, norte do estado do Paraná, Brasil. Possui uma área total de $2.226 \mathrm{~km}^{2}$, com $181,7 \mathrm{~km}^{2}$ de área construída, e um fragmento de Mata Atlântica conhecido como Horto Florestal, com área de 12 ha (SHIBATTA et al., 2009) (Figura 1). O Horto Florestal é composto por Floresta Estacional Semidecidual em diferentes estágios de sucessão ecológica (REIS et al., 2012). Nesse local, a flora é diversificada, proporcionando fonte de alimento para a quiropterofauna, na qual se incluem espécies de Moraceae, Piperaceae, Solanaceae e Cecropiaceae.

\section{Métodos de amostragem}

A amostragem foi realizada entre outubro de 2012 e setembro de 2013 , com quatro capturas mensais totalizando 48 noites de capturas. A metodologia da captura se deu mediante o uso de quatro redes de neblina (9 $\mathrm{m}$ de comprimento $\mathrm{x} 3 \mathrm{~m}$ de altura). As redes foram abertas ao crepúsculo e fechadas $6 \mathrm{~h}$ depois.

Os animais capturados foram mantidos em sacos de algodão por cerca de 30 minutos para obtenção das amostras fecais. Antes da soltura, as espécies às quais os exemplares capturados pertenciam foram determinadas com base em guias de identificação (MIRANDA et al., 2011; REIS et al., 2013). As sementes obtidas nas fezes dos morcegos foram acondicionadas em envelopes de papel manteiga devidamente identificados com informações da espécie, data e horário de coleta.

Para a verificação da composição da dieta de A. lituratus, os meses de coleta foram agrupados por estações do ano: primavera - outubro a dezembro; verão - janeiro a março; outono - abril a junho; e inverno julho a setembro. A identificação dos itens alimentares foi conduzida no Laboratório de Ecologia de Mamíferos, localizado no Centro de Ciências Biológicas (CCB) da

FIGURA 1: Localização do campus da Universidade Estadual de Londrina e dos pontos de amostragem no Horto florestal em relação ao município de Londrina, Paraná, Brasil.

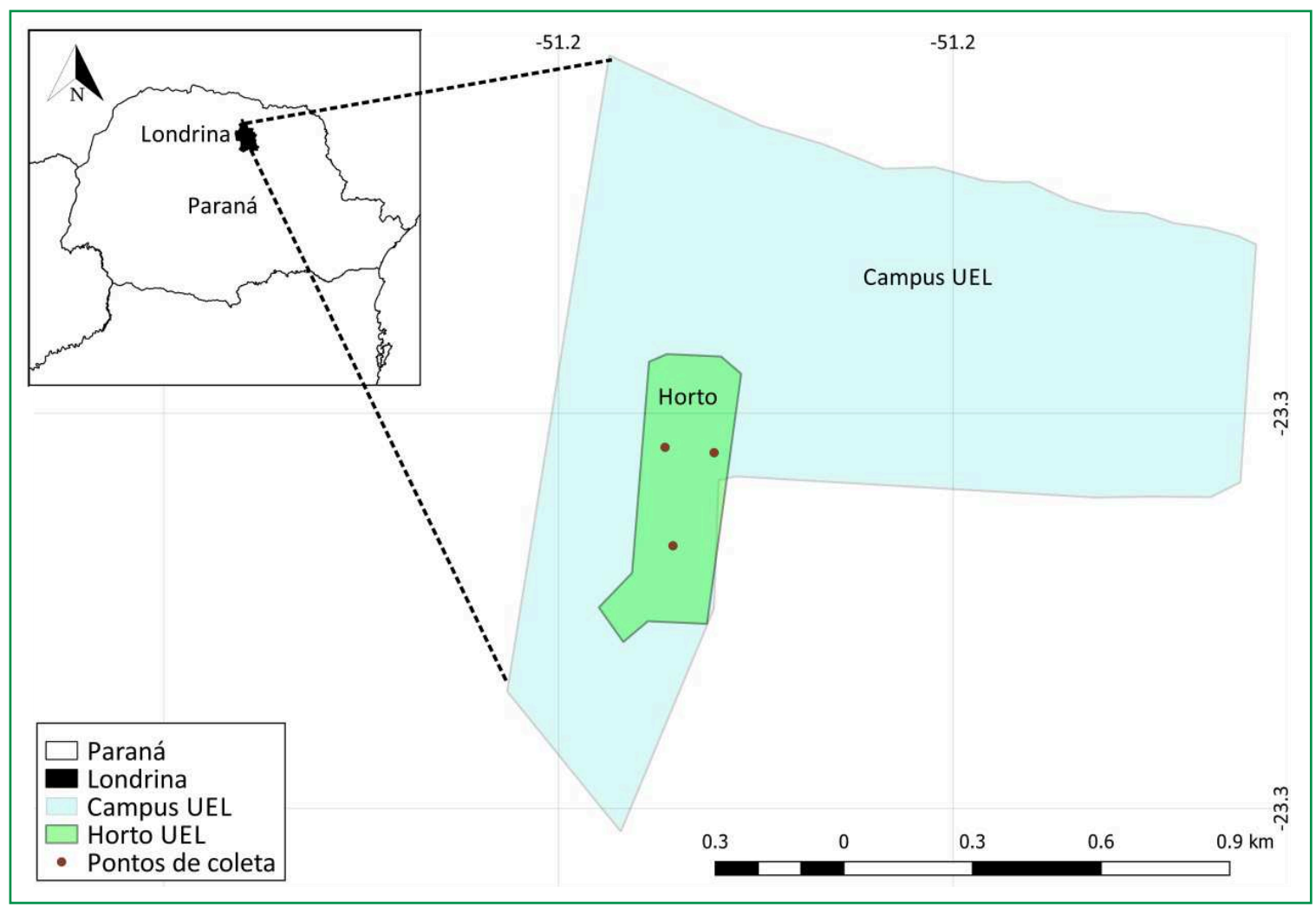


Universidade Estadual de Londrina (UEL). As sementes coletadas nas fezes dos animais foram identificadas com o auxílio de um microscópio estereoscópico, utilizando um banco de sementes disponível no laboratório e herbário da UEL.

\section{Análise dos dados}

Foi calculada a frequência de ocorrência (FO\%) para cada recurso alimentar de A. lituratus, expressa pelo número de amostras fecais que contém um ou mais recursos de cada espécie, em relação ao total de indivíduos analisados. Os dados de composição da dieta entre as estações do ano não apresentaram distribuição normal e foram avaliados pelo teste não paramétrico de Kruskal-Wallis, ao nível de significância de 5\%.

A amplitude de nicho trófico da espécie foi calculada pelo índice de Levin (HURLBERT, 1978): $\mathrm{B}_{\mathrm{i}}=\left[\left(\Sigma \mathrm{P}_{\mathrm{ij}}^{2}\right)^{-1}-1\right] /(\mathrm{n}-1)$, onde $B_{i}$ é o índice padronizado de amplitude de nicho, $P_{i j}$ é a proporção de recurso alimentar, $j$ na dieta da espécie; $i$ e $n$ representam o número total de recursos alimentares encontrados nas amostras. Para essa análise foi utilizada a contagem dos recursos alimentares da espécie (quantificação dos recursos). Esse índice assume que a amplitude da dieta pode ser estimada pela uniformidade na distribuição dos itens entre os diversos recursos alimentares (HURLBERT, 1978). O valor de B varia de 0 (quando a espécie consumiu principalmente um recurso alimentar) a 1 (quando a espécie consumiu todos os recursos em proporções semelhantes). Os valores de amplitude foram considerados altos quando $B>0,60$, moderados quando os valores de $B$ estiveram entre 0,20 e 0,60 e baixos quando $\mathrm{B}<0,20$.

\section{Resultados}

Foram capturados 86 indivíduos de A. lituratus e, a partir destes, 42 amostras fecais foram obtidas. Os recursos alimentares foram primariamente plantas, representadas por cinco famílias, cinco gêneros e doze espécies. Entre os itens contidos em amostras fecais, pedaços de tecido vegetal, fibras e sementes danificadas integram a categoria de itens não identificados (Figura 2).

FIGURA 2: Porcentagem dos itens vegetais encontrados nas amostras fecais de Artibeus lituratus coletadas entre outubro/2012 e setembro/2013 na Universidade Estadual de Londrina, Londrina, PR, Brasil. Barras verticais representam o desvio padrão.

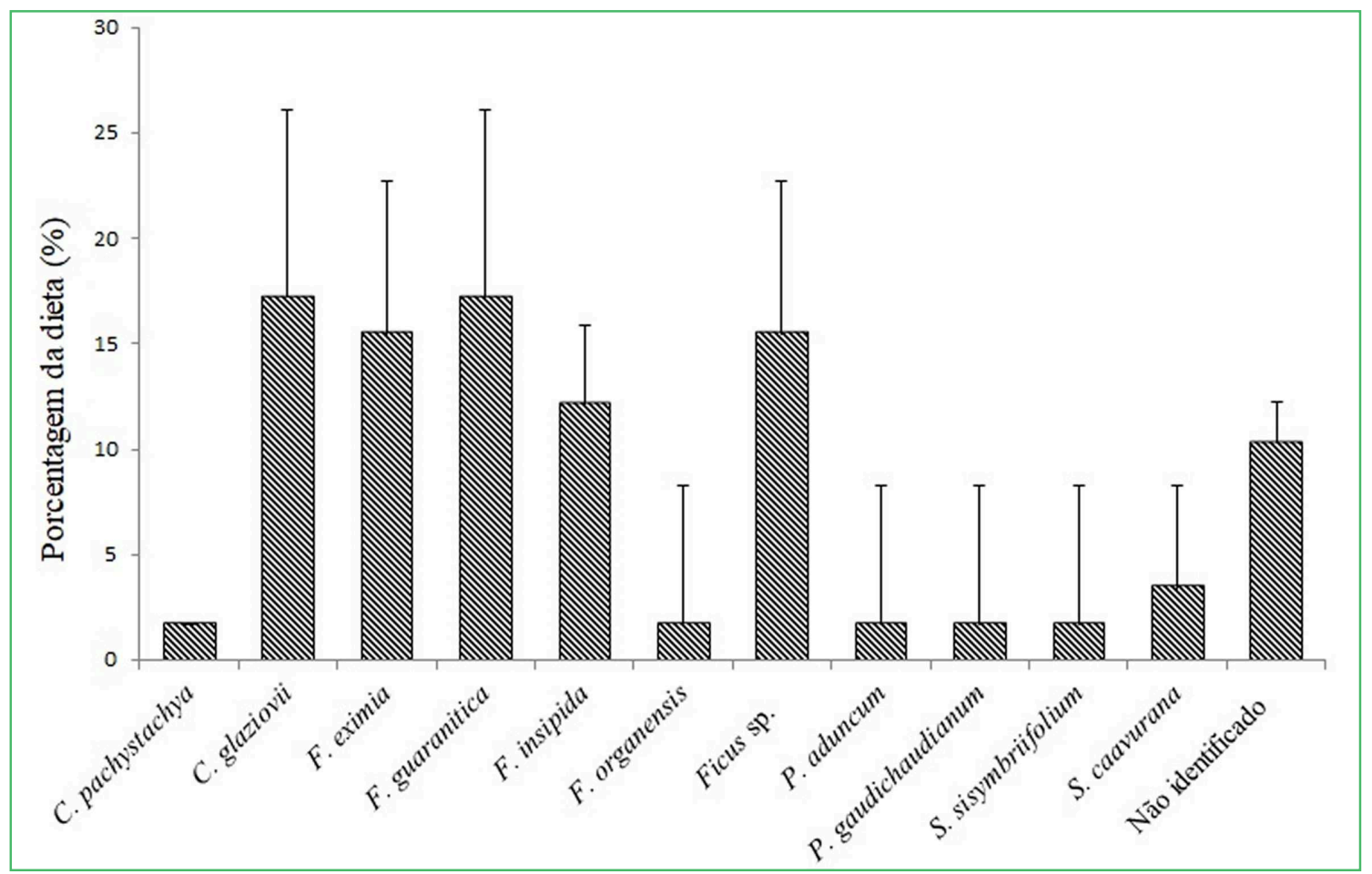


Ocorreram diferenças na quantidade de amostras fecais coletadas ao longo das estações bem como na quantidade de morcegos. Na primavera, 11 indivíduos foram capturados e oito amostras fecais foram coletadas $(\mathrm{FO}=19,05 \%)$, sendo identificadas sementes de Cecropia glaziovii Snethl., C. pachystachya Trécul., Ficus eximia Schott e F. guaranitica Chodat. No verão, 36 indivíduos foram capturados e 11 amostras fecais foram coletadas (FO $=26,19 \%)$ contendo Ficus eximia, F. guaranitica, Cecropia glaziovii, C. pachystachya, Solanum sisymbriifolium Lam. e Solanum caavurana Vell. Nesta mesma estação, ocorreram capturas de morcegos carregando frutos de Terminalia catappa Linn. Durante a estação do outono, 21 indivíduos foram capturados e coletadas 13 amostras fecais $(\mathrm{FO}=30,95 \%)$, compostas, principalmente, pelo gênero Ficus (Ficus sp., F. eximia, F. guaranitica, F. insipida Willd.) e também foram encontradas sementes de C. glaziovii, Piper aduncum L. e P. gaudichaudianum Kunth. No inverno, 18 indivíduos foram capturados e 10 amostras fecais foram coletadas (FO $=23,81 \%)$, compostas por sementes de Ficus sp., . eximia, F. guaranitica, F. insipida e F. organensis (Miq.) Miq. (Tabela 1).

Com a análise de amplitude de nicho trófico podese observar uma dieta de maior amplitude nos meses de março, agosto e setembro de 2013 (Figura 3), contudo, não se observou variação significativa entre os itens alimentares identificados ao longo das estações do ano $(\mathrm{H}=0,3002 ; p=0,96)$.

\section{Discussão}

A dieta de A. lituratus foi composta principalmente por espécies da família Cecropiaceae e Moraceae, resultado que corrobora outros estudos que investigaram a preferência alimentar desta espécie em outras regiões, como no Cerrado (SATO et al., 2008; MARTINS et al., 2014), no sudeste brasileiro (PASSOS; PASSAMI, 2003), em áreas litorâneas de Mata Atlântica do Sul do Brasil (PASSOS; GRACIOLLI, 2004), bem como em fragmentos paranaenses (BRITO et al., 2010). Apesar do presente estudo não testar a relação de preferência alimentar da

TABELA 1: Itens alimentares da dieta de $A$. lituratus ao longo das estações no Campus da Universidade Estadual de Londrina, Londrina, PR, Brasil. NT = Número total de itens; $\mathrm{N}=$ número de itens por estação; $\mathrm{FO} \%=$ frequência de ocorrência e desvio padrão.

\begin{tabular}{|c|c|c|c|c|c|c|c|c|c|}
\hline \multirow{2}{*}{ Itens alimentares } & \multirow{2}{*}{ NT } & \multicolumn{2}{|c|}{ Primavera } & \multicolumn{2}{|r|}{ Verão } & \multicolumn{2}{|r|}{ Outono } & \multicolumn{2}{|c|}{ Inverno } \\
\hline & & $\mathbf{N}$ & FO\% & $\mathbf{N}$ & FO\% & $\mathbf{N}$ & FO\% & $\mathbf{N}$ & FO\% \\
\hline Ficus sp. & 9 & 0 & 0 & 0 & 0 & 8 & $47,1 \pm 0,5$ & 1 & $7,7 \pm 0,3$ \\
\hline Ficus eximia Schott & 9 & 4 & $30,8 \pm 0,5$ & 2 & $11,76 \pm 0,4$ & 1 & $5,9 \pm 0,3$ & 2 & $15,4 \pm 0,4$ \\
\hline Ficus guaranitica Chodat & 10 & 3 & $23,1 \pm 0,5$ & 1 & $5,88 \pm 0,3$ & 3 & $17,6 \pm 0,5$ & 3 & $23,1 \pm 0,5$ \\
\hline Ficus insipida Willd. & 7 & 0 & 0 & 0 & 0 & 1 & $5,9 \pm 0,3$ & 6 & $46,2 \pm 0,5$ \\
\hline Ficus organensis (Miq.) Miq. & 1 & 0 & 0 & 0 & 0 & 0 & 0 & 1 & $7,7 \pm 0,3$ \\
\hline Cecropia pachystachya Trécul. & 1 & 1 & $7,7 \pm 0,3$ & 0 & 0 & 0 & 0 & 0 & 0 \\
\hline Cecropia glaziovii Snethl. & 10 & 5 & $38,5 \pm 0,5$ & 3 & $17.65 \pm 0,4$ & 2 & $11,8 \pm 0,4$ & 0 & 0 \\
\hline Solanum sisymbriifolium Lam. & 1 & 0 & 0 & 1 & $5,88 \pm 0,3$ & 0 & 0 & 0 & 0 \\
\hline Solanum caavurana Vell. & 2 & 0 & 0 & 2 & $11,76 \pm 0,4$ & 0 & 0 & 0 & 0 \\
\hline Piper aduncum $\mathrm{L}$. & 1 & 0 & 0 & 0 & 0 & 1 & $5,9 \pm 0,3$ & 0 & 0 \\
\hline Piper gaudichaudianum Kunth & 1 & 0 & 0 & 0 & 0 & 1 & $5,9 \pm 0,3$ & 0 & 0 \\
\hline Terminalia catappa Linn & 2 & 0 & 0 & 2 & $11,76 \pm 0,4$ & & & & \\
\hline 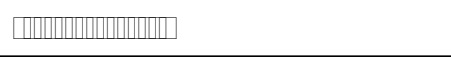 & 6 & 0 & 0 & 6 & $40 \pm 0,5$ & 0 & 0 & 0 & 0 \\
\hline Total & 60 & 13 & 100 & 15 & 100 & 17 & 100 & 13 & 100 \\
\hline
\end{tabular}


FIGURA 3: Amplitude de nicho trófico de A. lituratus no Campus da Universidade Estadual de Londrina, Londrina, PR, Brasil.

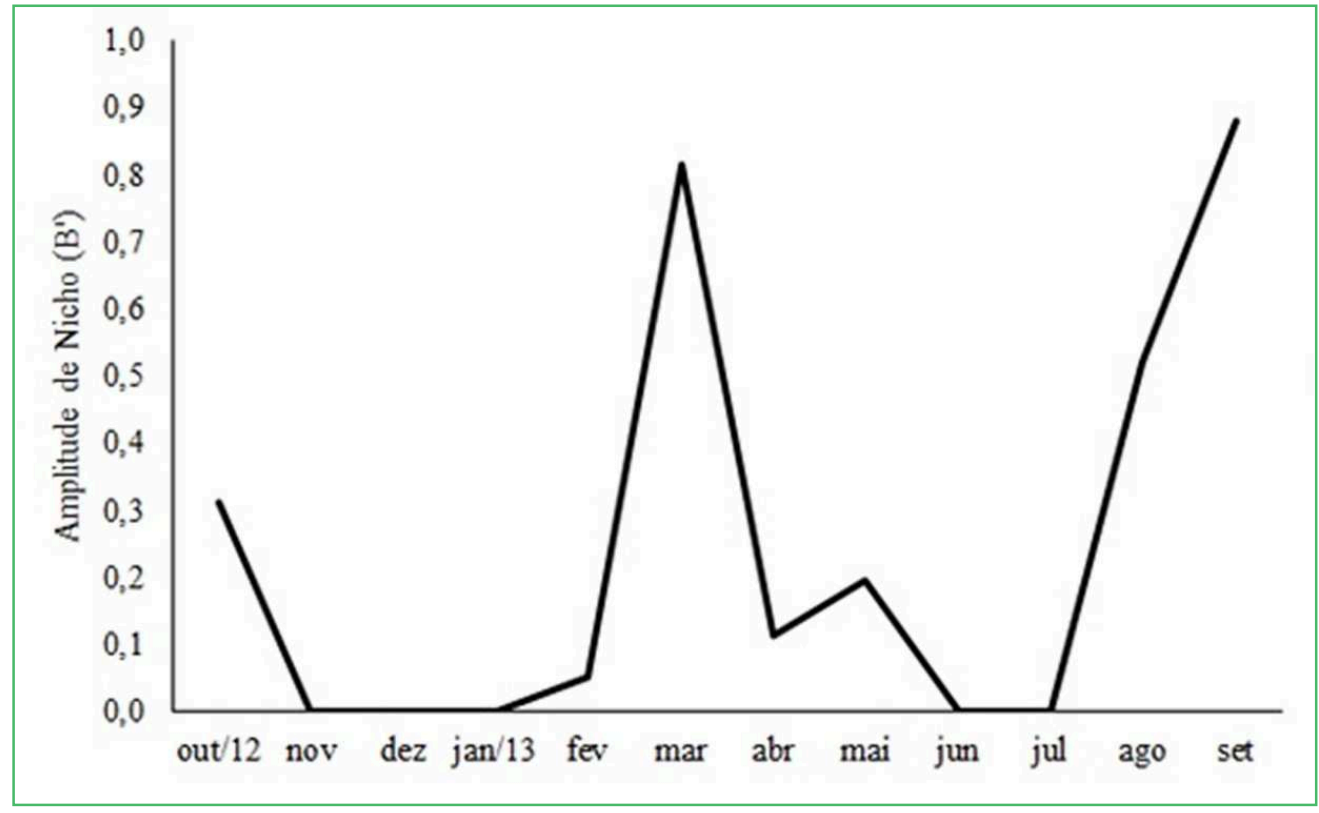

espécie, os dados reforçam o grau de especialização de $A$. lituratus em relação a frutos de Cecropiaceae e Moraceae (FLEMING, 1986).

Registrou-se também o consumo de frutos das famílias Solanaceae e Piperaceae, resultados similares aos de Muller e Reis (1992) em fragmentos florestais na região norte do Paraná, além da presença de outros recursos florais não identificados nas fezes analisadas. Alguns trabalhos demonstram que A. lituratus, mesmo em ambientes muito alterados, tem o potencial de utilizar várias fontes de recursos, desde que estes estejam disponíveis, reforçando o potencial dessa espécie como um filostomídeo frugívoro generalista e oportunista, que se adapta a condições de disponibilidade de recursos (GALETTI; MORELLATO, 1994; PASSOS; PASSAMI, 2003; REIS et al., 2013).

Alguns autores como Mello et al. (2005) sugerem que $A$. lituratus tem preferência por frutos maiores, pois lhe fornecem uma maior quantidade de polpa. Durante duas noites de coletas, foram encontrados frutos de $T$. catappa caídos na rede de neblina ao lado de indivíduos de A. lituratus. Frutos grandes, como de T. catappa, não são encontrados nas fezes dos animais, mas costumam ser levados para poleiros de alimentação (GALETTI et al., 1999).
Em nosso estudo encontramos nas fezes de $A$. lituratus sementes de S. sisymbriifolium F. insipida, $C$. pachystachya e C. glaziovii (Tabela 1). Recentemente, para a região do Parque Estadual do Godoy, em Londrina, Batista et al. (2017) demonstram que a dieta de $A$. lituratus é rica não apenas em carboidratos, mas também em lipídeos e proteínas. Frutos de todas as plantas registradas no presente estudo demonstraram conter em sua composição elevadas concentrações de carboidratos e lipídios e ainda pequenas concentrações de proteínas (BATISTA et al., 2017). Sendo assim, para uma espécie de grande porte, como A. lituratus, e que realiza vários incursos a diferentes árvores, o consumo de frutos de determinadas espécies de plantas fornecem energia, principalmente de carboidratos, que suprem as demandas de alta energia gasta durante a atividade de busca de alimento durante o voo (VOIGT et al., 2010; BATISTA et al., 2017).

Conforme mencionado, a área deste estudo é a de um pequeno fragmento de Floresta Estacional Semidecidual com diferentes estágios de sucessão, localizado em perímetro urbano. A sucessão é o principal mecanismo do processo de restauração e pode ser mediada por diferentes agentes bióticos e abióticos, interagindo em diferentes dimensões de nicho no tempo e espaço (YOUNG et al., 2005). 
O papel de morcegos para a restauração de áreas degradadas é conhecido na literatura, principalmente em regiões Neotropicais, onde são polinizadores e dispersores de diversas plantas (LOBOVA et al., 2009; KUNZ et al., 2011; BREDT et al., 2012). Martins et al. (2014) constataram em seu estudo um grande consumo de plantas pioneiras por filostomídeos. Em um fragmento florestal do Instituto São Vicente, em Campo Grande MS, A. lituratus apresentou preferências alimentares por frutos de Cecropia, Ficus e Piper, gêneros de espécies pioneiras (MARTINS et al., 2014).

As informações sobre a dieta de morcegos frugívoros são contribuições fundamentais para o entendimento da dinâmica e estrutura de comunidade de morcegos e de todo o ecossistema, possibilitando ferramentas para a conservação dessas áreas (BREDT et al., 2012). A continuidade do estudo nesta região utilizando outros métodos, como poleiros de alimentação, observações diretas, avaliação dos períodos de frutificação poderão dar suporte a programas de restauração em áreas degradadas e pequenos fragmentos florestais.

\section{Referências}

BATISTA, C. B.; REIS, N. R.; REZENDE, M. I. Nutritional content of bat-consumed fruits in a forest fragment in Southern Brazil. Brazilian Journal of Biology, São Carlos, v. 77, n. 2, p. 244-250, 2017.

BREDT, A.; UIEDA, W.; PEDRO, W. A. Plantas e morcegos: na recuperação de áreas degradadas e na paisagem urbanas. Brasília: Rede de Sementes do Cerrado, 2012. 273 p.

BRITO, J. E. C.; GAZARANI, J.; ZAWADZKI, C. H. Abundância e frugivoria da quiropterofauna (Mammalia, Chiroptera) de um fragmento no noroeste do estado do Paraná, Brasil. Acta Scientiarum Biological Sciences, Maringá, v. 32, n. 3, p. 265-271, 2010.

ESTRADA, A.; COATES-ESTRADA, R. Bats in continuous forest, forest fragments and in an agricultural mosaic habitat-island at Los Tuxtlas, Mexico. Biological Conservation, Boston, v. 103, n. 2, p. 237-245, 2002.

FLEMING, T. H. Opportunism versus specialization: evolution of feeding strategies in frugivorous bats. In: ESTRADA, A.; FLEMING, T. H. (Ed.). Frugivores and seed dispersal. Stanford: Springer Netherlands, 1986. p. 105-118.

GALETTI, M.; MORELLATO, L. P. C. Diet of the large fruit-eating bat Artibeus lituratus in a forest fragment in Brazil. Mammalia, Paris, v. 58, n. 4, p. 661-665, 1994.

GALETTI, M.; ZIPARRO, V. B.; MORELLATO, L. P. C. Fruiting phenology and frugivory on the palm Euterpe edulis in a lowland Atlantic Forest of Brazil. Ecotropica, Frankfurt, v. 5, p. 115-122, 1999.
GALINDO-GONZÁLEZ, J.; GUEVARA, S.; SOSA, V. J. Bat and bird, generated seed rains at isolated trees in pastures in a tropical rainforest. Conservation Biology, San Francisco, v. 14, n. 6, p. 1693-1703, 2000.

HURLBERT, S. H. The measurement of niche overlap and some relatives. Ecology, New York, v. 59, n. 1, p. 67-77, 1978.

KUNZ, T. H.; TORRES, E. B.; BAUER, D.; LOBOVA, T.; FLEMING, T. H. Ecosystem services provided by bats. Annals of the New York Academy of Sciences, New York, v. 1223, n. 1, p. 1-38, 2011.

LOBOVA, T. A.; CULLEN, K. G.; SCOTT A. M. Seed dispersal by bats in the Neotropics. New York: New York Botanical Garden Press, 2009. 465 p.

MARTINS, M. P.; TORRES, J. M.; ANJOS, E. A. C. Dieta de morcegos filostomídeos (Mammalia, Chiroptera, Phyllostomidae) em fragmento urbano no Instituto São Vicente, Campo Grande, Mato Grosso do Sul. Papeis Avulsos de Zoologia, São Paulo, v. 54, n. 20, p. 299-305, 2014.

MELLO, M. A. R.; LEINER, N. A.; GUIMARÃES, R.; JORDANO, P. Size based fruit selection of Calophyllum brasiliense (Clusiaceae) by bats of the genus Artibeus (Phyllostomidae) in a Restinga area, southeastern Brazil. Acta Chiropterologica, Warsawa, v. 7, n. 1, p. 179-182, 2005.

MIRANDA, J. M. D.; BERNARDI, I. P.; PASSOS, F. C. Chave ilustrada para determinação dos morcegos da região sul o Brasil. Curitiba: Universidade Federal de Curitiba, 2011. 56 p.

MULLER, M. F.; REIS, N. R. Partição de recursos alimentares entre quatro espécies de morcegos frugívoros (Chiroptera, Phyllostomidae). Revista Brasileira de Zoologia, Curitiba, v. 9, n. 4, p. 345-355, 1992.

PASSOS, F. C.; GRACIOLLI, G. Observações da dieta de Artibeus lituratus (Olfers) (Chiroptera, Phyllostomidae) em duas áreas do sul do Brasil. Revista Brasileira de Zoologia, Curitiba, v. 21, n. 3, p. 487-489, 2004.

PASSOS, J. G.; PASSAMANI, M. Artibeus lituratus (Chiroptera, Phyllostomidae): biologia e dispersão de sementes no Parque do Museu de Biologia Prof. Mello Leitão, Santa Teresa (ES). Natureza On Line, Santa Teresa, v, 1, n. 1, p. 1-6, 2003.

PERACCHI, A. P.; LIMA, I. P. Chave dicotômica para as espécies de morcegos que ocorrem no Brasil. In: REIS, N. R.; PERACCHI, A. P.; BATISTA, C. B.; LIMA, I. P.; PEREIRA, A. D. (Ed.). História natural dos morcegos brasileiros: chave de identificação de espécies. Rio de Janeiro: Technical Books Editora, 2017. p. 21-62.

PEREIRA, A. D.; BORDIGNON, M. O.; TAVARES, V. C. Subfamília Stenodermatinae Gervais, 1856. In: REIS, N. R.; PERACCHI, A. P.; BATISTA, C. B.; LIMA, I. P.; PEREIRA, A. D. (Ed.). História natural dos morcegos brasileiros: chave de identificação de espécies. Rio de Janeiro: Technical Books Editora, 2017. p. 208-247.

REIS, N. R.; BARBIERI, M. L. S.; LIMA, I. P.; PERACHI, A. L. O que é melhor para manter a riqueza de espécies de morcegos (Mammalia, Chiroptera): um fragmento florestal grande ou vários fragmentos de pequeno tamanho? Revista Brasileira de Zoologia, Curitiba, v. 20, n. 2, p. 225-230, 2003. 
REIS, N. R.; FREGONEZI, M. N.; PERACCHI, A. L.; SHIBATTA, O. A. Morcegos do Brasil: guia de campo. Rio de Janeiro: Technical Books, 2013, 252 p.

REIS, N. R.; GALLO, P.; PERACCHI, A. L. Sensitivity of populations of bats (Mammalia: Chiroptera) in relation to human development in northern Paraná, southern Brazil. Brazilian Journal of Biology, São Carlos, v. 72, n. 3, p. 511-518, 2012.

REIS, N. R.; PERACCHI, A. L.; BATISTA, C. B.; LIMA, I. P.; PEREIRA, A. D. História natural dos morcegos brasileiros: chave de identificação de espécies. Rio de Janeiro: Technical Books, 2017. $416 \mathrm{p}$.

REIS, N. R.; PERACCHI, A. L.; SANTOS, G. A. S. D. Ecologia de morcegos. Londrina: Technical Books, 2008. 148 p.

SATO, T. M.; PASSOS, F. C.; NOGUEIRA, A. C. Frugivoria de morcegos (Mammalia, Chiroptera) em Cecropia pachystachya (Urticaceae) e seus efeitos na germinação das sementes. Papéis Avulsos de Zoologia, São Paulo, v. 48, n. 3. p. 19-26, 2008.

SAZIMA, M.; BUZATO, S.; SAZIMA, I. Dyssochroma viridiflorum (Solanaceae): a reproductively bat-dependent epiphyte from the Atlantic Rainforest in Brazil. Annals of Botany, Oxford, v. 92, n. 5, p. 725-730, 2003.
SHIBATTA, O. A.; GALVES, W.; CARMO, W. P. D.; LIMA, I. P.; LOPES, E. V.; MACHADO, R. A. A. Fauna de vertebrados do campus da Universidade Estadual de Londrina, região norte do estado do Paraná, Brasil. Semina: Ciências Biológicas e da Saúde, Londrina, v. 30, n. 1, p. 3-26. 2009.

SOS MATA ATLANTICA; INPE - INSTITUTO NACIONAL DE PESQUISAS ESPACIAIS. Atlas dos remanescentes florestais da Mata Atlântica - Período 2016-2017. Relatório Técnico, São Paulo, 2018. 66 p.

TERBORGH, J.; WRIGHT, J. Effects of mammalian herbivores on plant recruitment in two Neotropical Forests. Ecology, New York, v. 75, n. 6, p. 1829-1833, 1994.

THIES, W.; KALKO, E. K. V. Phenology of neotropical pepper plants (Piperaceae) and their association with their main dispersers, two short-tailed fruit bats, Carollia perspicillata and C. castanea (Phyllostomidae). Oikos, Copenhagen, v. 104, n. 2, p. 362-376, 2004.

VOIGT, C. C.; SÖRGEL, K.; DECHMANN, D. K. N. Refueling while flying: foraging bats combust food rapidly and directly to power flight. Ecology, New York, v. 91, n. 10, p. 2908-2917, 2010.

YOUNG, T. P.; PETERSEN, D. A.; CLARY, J. J. The ecology of restoration: historical links, emerging issues and unexplored realms. Ecology Letters, Montpellier, v. 8, n. 6, p. 662-673, 2005. 